\title{
Construction of a Fast Diode-Laser based Calibration System for Time-of-Flight Systems
}

\section{Bonesini*; R. Bertoni}

Sezione INFN Milano Bicocca, Dipartimento di Fisica G. Occhialini, Piazza Scienza 3, Milano, Italy

\section{A. de Bari, M. Rossella}

Sezione INFN Pavia and Dipartimento di Fisica, Università di Pavia, via Bassi 6, Pavia, Italy

\begin{abstract}
For the calibration and monitoring of scintillator based time-of-flight systems, fast laser pulses, delivered to the individual channels, may be used. Low-cost laser diodes with FWHM time resolution in the range 30-50 ps may be chosen as light source. The problem is their low output power, as compared to traditional solutions as expensive duplicated Nd-Yag lasers. The tests done to assemble a prototype system based on optical switches and fiber fused splitters will be summarized, as well as the ones on the full-scale prototype. The main concern was the tight power budget of the system.
\end{abstract}

The European Physical Society Conference on High Energy Physics 18-24 July, 2013

Stockholm, Sweden

\footnotetext{
* Speaker.

†E-mail: maurizio.bonesini@mib.infn.it
} 

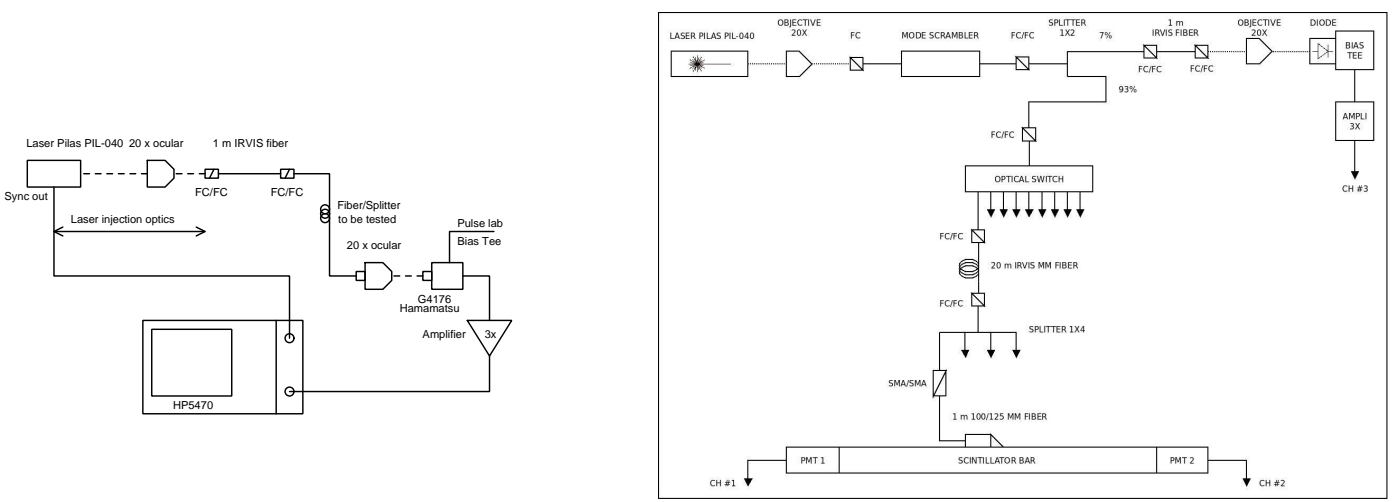

Figure 1: Left: experimental setup for test of single optical components. In the attenuation studies the Hamamatsu photodetector+HP54750 sampling scope was replaced by a powermeter. Right: schematic layout of the prototype calibration system.

Fast scintillator based time-of-flight (TOF) detectors may be made with single counters read by photomultipliers (PMTs) at both ends. From each PMT a timing measurement $\Delta t_{i}\left(t_{0}, \delta_{i}\right)$, that depends on the particle crossing time $t_{0}$, is obtained. The individual time delays $\delta_{i}$ depend on cable lengths, electronic delays, ... They may drift during the data-taking, due to temperatures excursions and other effects [1]. As an example, RG58 (RG213) signal cables may have time variation up to 95 $\mathrm{ppm} /{ }^{\circ} \mathrm{C}\left(30 \mathrm{ppm} /{ }^{\circ} \mathrm{C}\right)[2]$. With typical delays around $100 \mathrm{~ns}$, time drift of the order of $9(3) \mathrm{ps} /{ }^{\circ} \mathrm{C}$ may be reached for RG58 (RG213) cables. This must be compared with a TOF detctor resolution $\left(\sigma_{t}\right)$ in the range 50-100 ps. Detector timing calibration in a time-of-flight system means the precise determination of delays $\delta_{i}(t)$ at an initial time $t_{0}$ and their monitoring along the data-taking. This may be done with cosmic rays or impinging particles, if available, or by delivering a fast laser calibration pulse to each channel. The use of low-cost laser-diode systems with time resolutions in the range 30-50 ps (FWHM), but unfortunately also limited power, has been proposed. This limitation imposes severe constraints on the optical delivery system to individual channels.

A laser diode calibration system may be built up from optical switches that direct the input laser pulse on one of $N$ output channels, fused fiber splitters $1 \times N$ that divide the input laser pulse to $N$ output channels and fiber patch cables for connections between the previous items. All items are easily available at Telecom wavelenghts $(\sim 1200 \mathrm{~nm})$, but difficult to find for the visible range.

The laboratory setup used for optical elements characterization is shown in figure 1. Light from a fast Pilas 040 laser from Advanced Laser Systems (FWHM $\sim 30$ ps, peak power $1 \mathrm{~W}, \lambda \sim 405$ $\mathrm{nm}$ ) is injected into a MM Mode Scrambler (from Arden Photonicd Ltd.) using a Newport $20 \mathrm{X}$ objective. Fine alignment for the injection is obtained via a $x, y, z$ Newport micrometric stage. After the last optical element under test, the ouput light pulse is focalized, via another 20X objective put on a micrometric $x, y, z$ flexure stage, on a fast MSM Hamamatsu G4176 photodetector (with 30 ps rise and fall time), powered by a broadband (10 GHz) bias tee (Model 5550B from Picosecond Pulse Lab). The amplified signal is then fed into a $20 \mathrm{GHz}$ HP 54750 sampling scope.

To guarantee an optimal and simple injection of the light from the laser source, large core multimode fibers (MM) are to be preferred to small core single mode (SM) fibers. The problem is that MM fibers have remarkable intermodal dispersion. Results on fiber tests are summarized in table 1 for $50 \mu \mathrm{m}$ and $100 \mu \mathrm{m}$ core MM available fibers. Fiber patches up to 10-15 meters 
introduce a dispersion less than $10-15 \mathrm{ps}\left(\sigma_{t}\right)$.

Table 1: Attenuation and timing properties of measured MM fibers.

\begin{tabular}{|c|c|c|c|}
\hline fiber type & & Attenuation $(\mathrm{dB} / \mathrm{m})$ & Increase in $\sigma_{t}(\mathrm{ps} / \mathrm{m})$ \\
\hline IRVIS OZ/Optics & MM 50/125 & 0.054 & 1.0 \\
Corning & MM 50/125 & 0.087 & 1.5 \\
Thorlabs SFS & MM 50/125 & 0.072 & 1.4 \\
Thorlabs AFS & MM 50/125 & 0.23 & 2.4 \\
Thorlabs SFS & MM 100/125 & - & 2.9 \\
\hline
\end{tabular}

A custom-made MM optical switch $1 \times 9$ by (Model F-109-05 from PiezoJena) and several custom-made MM optical splitters $1 \times 2,1 \times 4,1 \times 8$ by OZ/Optics and Lightel were tested. After the mode scrambler, the measured FWHM of the laser signal increased from $80.47 \pm 0.61 \mathrm{ps}$ to typical values around $83.14 \pm 0.46 \mathrm{ps}$ inserting the optical switch. The output signal variation from channel to channel was within $1 \%$, with a cross-talk $\sim 2 \%$ and an insertion loss $\sim 40 \%$. Similar measurements were done inserting optical fiber splitters $(1 \times 4$ or $1 \times 8)$ in place of the optical switch. We see a relevant insertion loss at $400 \mathrm{~nm}(\sim 3 \mathrm{~dB})$, a dispersion in the splitting ratio $\sim 10-15 \%$ (rms/average) and an increase of the time spread of the input signal $\leq 1 \mathrm{ps}\left(\sigma_{t}\right)$. In conclusion, the tested optical components seem suitable to assemble the pulse delivery system for a diode-laser based calibration system.

A prototype calibration system has been assembled from the previous optical elements. In its present configuration, up to 36 (72) individual channels (using $1 \times 4$ or $1 \times 8$ splitters) may be calibrated. It may easily extended to configurations with up to 100-200 channels. Figure 1 shows the setup presently assembled in laboratory, where the light from one $1 \times 4$ splitter is injected, by means of a reflection prism, into the center of the scintillation counter to be calibrated. The PMT signals were acquired with a VME system, based on the CAEN V2718 interface, and splitted by a 50\% passive splitter to a CAEN V792 QADC and a CAEN V1290 TDC, after a leading edge discriminator. We see no appreciable difference in the L-R TDC difference for a laser signal directly sent to the scintillation counter via the reflection prism and the $1 \mathrm{~m} \mathrm{MM} \mathrm{100/125} \mu \mathrm{m}$ fiber inserted into it and the signal delivered after full the calibration system. TDC signals are computed as differences between the L/R PMT signal (ch 1 or 2 in figure 1) and a reference signal (ch 3 in figure 1) from a fast Thorlabs DET02A photodiode (rise time $50 \mathrm{ps}$ and fall time $150 \mathrm{ps}$ ). Care has been put to have the intensity of the direct signal comparable to the one after the calibration system, by using fiber optical attenuators.

We thus conclude that such a calibration system may be used for fast scintillator based TOF system, with up to 100-200 channels and timing resolutions in the range 50-100 ps, such as the one developed for the MICE experiment at RAL [3]

\section{References}

[1] M.Bonesini et al. IEEE Trans Nucl. Science NS-50(4) 20031053

[2] M. Baldo Ceolin et al. NIM A532 (2004) 548

[3] R. Bertoni et al. NIM A615 (2010) 14 